\title{
Symbolic Reach-Avoid Control of Multi-Agent Systems
}

\author{
Rupak Majumdar \\ MPI-SWS, Germany. \\ rupak@mpi-sws.org
}

\author{
Kaushik Mallik \\ MPI-SWS, Germany. \\ kmallik@mpi-sws.org
}

\author{
Mahmoud Salamati \\ MPI-SWS, Germany. \\ msalamati@mpi-sws.org
}

\author{
Sadegh Soudjani \\ Newcastle University, UK. \\ sadegh.soudjani@ncl.ac.uk
}

\author{
Mehrdad Zareian \\ MPI-SWS, Germany. \\ mzareian@mpi-sws.org
}

\begin{abstract}
We consider the decentralized controller synthesis problem for multi-agent systems with global reach-avoid specifications. Each agent is modeled as a nonlinear dynamical system with disturbances. The objective is to synthesize local feedback controllers that guarantee that the overall multi-agent system meets the global specification despite the influence of disturbances. On the one hand, existing techniques based on planning or trajectory optimization usually ignore the effects of disturbances and produce open-loop nominal trajectories that are not generally sufficient in the presence of disturbances. On the other hand, techniques based on formal synthesis, which guarantee satisfaction of temporal specifications, do not scale as the number of agents increases.

We address these limitations by proposing a two-level solution approach that combines fast global nominal trajectory generation and local application of formal synthesis. At the top level, we ignore the effect of disturbances and obtain a joint open-loop plan for the system using a fast trajectory optimizer. At the lower level, we use abstraction-based controller design to synthesize a set of decentralized feedback controllers that track the high level plan against worst-case disturbances, thus ensuring satisfaction of the global specification.

We provide the implementation of our approach in an opensource tool called GAMARA. We demonstrate the effectiveness of GAMARA on several multi-robot examples using two particular classes of control specifications. In the first type, we assume that the robots need to fulfill their own reach-avoid tasks while avoiding collision with each other. In the second type, we require the robots to fulfill reach-avoid tasks while maintaining certain formation constraints. The experiments show that GAMARA produces formally guaranteed feedback controllers while scaling to many robots. In contrast, nominal open-loop controllers do not guarantee the satisfaction of the specification, and global formal approaches run out of memory before synthesizing a controller.
\end{abstract}

\section{CCS CONCEPTS}

\section{- Computer systems organization $\rightarrow$ Robotic control.}

Permission to make digital or hard copies of part or all of this work for personal or classroom use is granted without fee provided that copies are not made or distributed for profit or commercial advantage and that copies bear this notice and the full citation on the first page. Copyrights for third-party components of this work must be honored.

For all other uses, contact the owner/author(s).

ICCPS '21, May 19-21, 2021, Nashville, TN, USA

(c) 2021 Copyright held by the owner/author(s).

ACM ISBN 978-1-4503-8353-0/21/05.

https://doi.org/10.1145/3450267.3450548

\section{ACM Reference Format:}

Rupak Majumdar, Kaushik Mallik, Mahmoud Salamati, Sadegh Soudjani, and Mehrdad Zareian. 2021. Symbolic Reach-Avoid Control of Multi-Agent Systems. In ACM/IEEE 12th International Conference on Cyber-Physical Systems (with CPS-IoT Week 2021) (ICCPS '21), May 19-21, 2021, Nashville, TN, USA. ACM, New York, NY, USA, 12 pages. https://doi.org/10.1145/3450267. 3450548

\section{INTRODUCTION}

We consider the decentralized feedback controller synthesis problem for multi-agent, nonlinear systems against temporal reach-avoid specifications. By multi-agent, we mean that the systems under study are composed of a number of concurrently executing components. Each component is modeled as a possibly nonlinear dynamical system that evolves under the influence of a control as well as an environmental disturbance. Our specifications require that the global state of the system eventually reaches a target while avoiding certain bad states along the way. While the dynamics of each component is independent of the others, the overall trajectories are coupled by the global specification. Decentralized means that we require a solution in which each component has a local feedback controller that sees only the local state, but the combination of all the closed loops satisfy the global specification. Above all, our goal is to ensure the resulting controllers are provably correct against the worst-case model of disturbances.

Such multi-agent control problems are ubiquitous in the domain of robotics, where a number of (possibly heterogeneous) mobile robots move concurrently in a shared workspace. A global specification can ask, for example, that a set of robots be able to reach certain locations while avoiding collisions among themselves or with obstacles in the environment, or that a set of drones fly in formation while reaching a target. Indeed, automatic generation of decentralized controllers is a classical problem in robotics, artificial intelligence, and control theory, and there is an enormous literature on the subject-too many to enumerate-across these disciplines.

Despite the large body of research, few techniques today can handle all our desiderata. Multi-agent planning algorithms, such as (hybrid variants of) $A^{*}$ search, scale to large systems but typically either disregard or simplify the underlying dynamics and work with geometric or discrete models, or disregard the effect of disturbances or nonlinear dynamics. Most planning and trajectory optimization techniques handle the nominal dynamics, i.e., the dynamics free of disturbances, and construct open-loop controllers. However, the open-loop behaviors do not guarantee satisfaction of the specifications in the presence of disturbances. On the other hand, correct-by-construction controller synthesis techniques from 


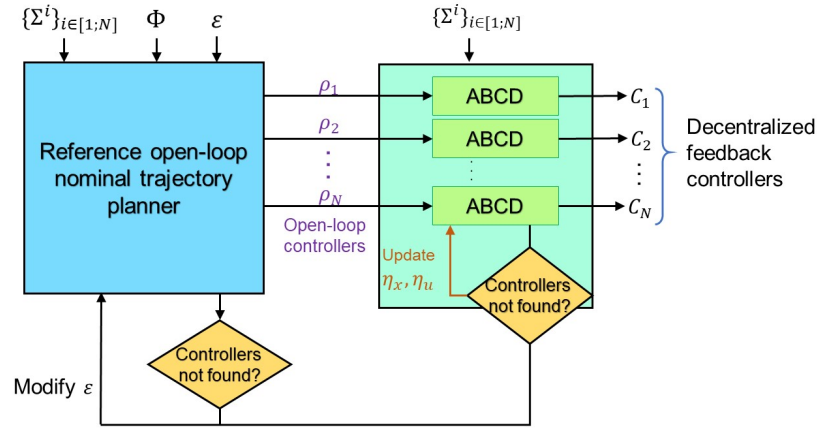

Figure 1: Overall algorithm: The blue block on the left (centrally) computes a joint open-loop nominal trajectory for the overall system. The green block on the right computes decentralized controllers for tracking the nominal trajectory using Abstraction Based Controller Design (ABCD). $\left\{\Sigma^{i}\right\}_{i \in[1 ; N]}$ is a set of $N$ agents, $\Phi$ is a global reach-avoid specification, $\varepsilon$ is a robustness margin, $\rho_{1}, \ldots, \rho_{N}$ are the local projections of the nominal trajectory, $\eta_{x}, \eta_{u}$ are parameters used in $\mathrm{ABCD}$, and $C_{1}, \ldots, C_{N}$ are the sought local feedback controllers for the individual agents.

control theory, such as abstraction-based control design (ABCD) or Hamilton-Jacobi techniques, handle precise models of nonlinear dynamics and the effects of disturbance, but are difficult to scale beyond about 10 dimensions.

In this paper, we provide a simple but effective combined approach. We use a global planning approach for nominal trajectory generation and a local correct-by-construction feedback controller synthesis approach for guaranteed adherence to specification for each component in the presence of disturbances. Fig. 1 shows the overall algorithm. In the first step, given a set of control systems, one for each component, and a reach-avoid specification on the global state space, we use a trajectory planner to find a nominal open-loop controller for the global system. The trajectory planner ignores the effect of disturbances, but takes a robustness parameter $\varepsilon$. The role of the robustness parameter is to ensure that the specification is robustly satisfied: Every trajectory within an $\varepsilon$-tube of the open-loop trajectory also satisfies the specification.

Next, we project the unique open-loop trajectory produced by the open-loop controller on to a nominal trajectory for each individual component. The robustness of the trajectory means that there is a tube around each nominal trajectory. In a second step, we solve a number of local guaranteed tracking control problems, where we synthesize correct-by-construction controllers whose objective is to track the nominal trajectory while staying within the tube. The overall algorithm is more scalable and guarantees satisfaction of the global specification.

We show empirically that our algorithm is able to generate provably correct feedback controllers for many systems for which neither technique is individually effective. Of course, since we decompose the problem, it is possible that there is no controller for a particular choice of the robustness parameter, or indeed, for other

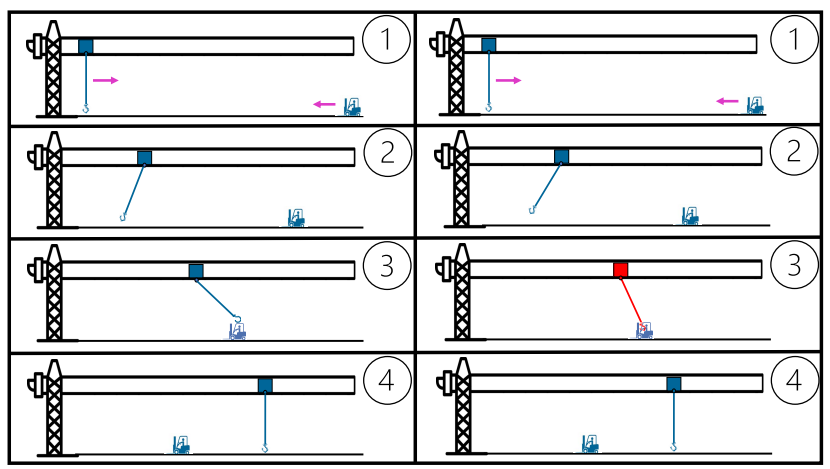

Figure 2: Illustration of the trajectories generated by the open-loop controller for the crane and vehicle example under disturbance-free (left) and perturbed (right) situations.

parameters used by the individual tools. In that case, there is an outer loop that searches through the parameter space.

We have implemented our approach in an open-source tool called GAMARA (stands for GuAranteed Multi-Agent Reach-Avoid control) by combining the following two tools: the ALTRO open-loop trajectory planner [15] and the SCOTS correct-by-construction controller synthesis tool [31]. ALTRO is a state-of-the-art trajectory planning tool based on optimal control. It handles nonlinear dynamics and scales to large dimensions, but ignores disturbances or modeling uncertainties. SCOTS implements a highly parallelizable ABCD algorithm that generates a feedback controller for satisfying temporal specification.

We empirically evaluate GAMARA on a number of multi-robot benchmarks, including a coordinated reach-avoid problem for ground robots, a formation control problem for drones, and a lane merging scenario for autonomous vehicles. In each case, we demonstrate that GAMARA can find decentralized and correct controllers within reasonable time and memory bounds.

Fig. 2 shows a concrete multi-robot reach-avoid scenario with an overhead crane hanging from a trolley along a horizontal rail and a cart that drives underneath the crane in the same horizontal axis. The goal is to move the crane and the vehicle such that they do not collide. Fig. 2 (left) shows an animation of a possible open-loop behavior from an initial configuration (Frame 1) to a final one (Frame 4), where the crane and the vehicle have crossed each other. The trajectory is generated by accelerating the trolley, causing the crane to swing up and thus creating enough space for the vehicle to pass. Unfortunately, in the presence of disturbances, such as wind or a slippery floor, a trajectory may not be free of collisions: the same open-loop behavior can cause a collision (Fig. 2 right). Instead, GAMARA computes a global robust trajectory for the system; the robustness parameter ensures a "wider berth." The global trajectory is projected to the crane and the vehicle, and we compute guaranteed tracking controllers that ensure there is no collision despite the disturbances. In our experiments, planning with ALTRO took less than a second and feedback controller synthesis with SCOTS about 10 minutes. At the same time, a global approach to find a correct solution does not scale. The global state space is $10^{10}$ times larger and SCOTS timed out with $1.5 \mathrm{~TB}$ of memory. 
Related Work The field of multi-agent planning is too large for a comprehensive survey; we point to the text books $[8,20,21,32]$ for an introduction. We categorize closely related work into (1) those combining planning and tracking controller synthesis, (2) those addressing formal multi-agent controller synthesis, and (3) those combining (1) and (2). We provide a survey of these categories.

Combining planning and tracking. Techniques combining high-level planning and low-level tracking are a staple of classical planning and control. More recently, several techniques consider the problem of formal guarantees for such planners. Existing works differ in the dynamics that they can handle (e.g., linear or nonlinear), considered class of specifications, including disturbances, and scalability. A common approach is to perform the high-level planning over a lower dimensional model and then use Sum-of-Squares programming (SOS), Hamilton Jacobi (HJ), or satisfiability modulo convex programming $(\mathrm{SMC})$ to obtain a low-level controller ensuring a bounded error between the two models [14, 24, 27, 35].

In contrast to $[14,35]$, our method does not require finding a linear mapping between the low and high dimensional models. Meyer et al. [24] considered reach-avoid problems for perturbed non-linear control affine systems. They create a lower dimensional model and use SOS programming to compute a controller ensuring a bounded error between the two models. Then, they use ABCD to compute a controller for the low-order model while taking the error into account. While their method can provide guarantee against worst-case disturbances, it is not clear if SOS always scales to the higher dimensions.

Nilsson et al. [27] provide a method that decomposes the state space into a lower-order planning space, and a higher-order internal dynamics space, so that fast planning and accurate tracking can be achieved using a set of control barrier functions computed based on SOS. Despite providing guarantees for the worst-case bounded disturbances, their method is not capable of solving reach-avoid tasks which involve dynamic obstacles as in the multi-agent case. While we have chosen SCOTS since the underlying algorithm can be effectively parallelized [18], in principle, we could also use SOS, $\mathrm{HJ}$, or SMC approaches.

Other works only consider special classes of models such as linear [10, 30, 39], disturbance-free [11, 36, 38], or finite transition systems [42]. In contrast, our method supports arbitrary nonlinear dynamics and provides a guarantee against worst-case bounded disturbances.

Formal multi-agent synthesis. Chen et al. [7] provide a method, using control barrier functions, that requires some form of inter-robot communication and does not consider external disturbances. Sahin et al. [34] propose a method that requires the group of robots to be homogeneous. There are methods which do not consider external disturbances and do not provide formal guarantees [16].

Combinations. Alonso-Mora et al. [1] provide a method for formation control of a group of communicating homogeneous robots. They first synthesize a nominal controller using a fast randomized geometric planning method, namely RRT, and then use optimal control to track the obtained nominal solution. Unlike us, they neither consider external disturbances nor provide formal guarantees. Pant et al. [28] have studied multi-quadrotor missions with signal temporal logic (STL) specifications. They find the reference
Table 1: Features of the publicly available tools compared to GAMARA. Note that some of these tools can handle richer classes of specifications, compared to the reach-avoid problem handled by GAMARA.

\begin{tabular}{|c|c|c|c|}
\hline Tool name & 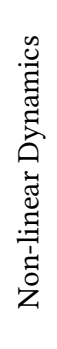 & 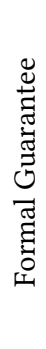 & 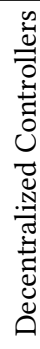 \\
\hline GAMARA & $\checkmark$ & $\checkmark$ & $\checkmark$ \\
\hline SCOTS [31] & $\checkmark$ & $\checkmark$ & \\
\hline ALTRO [15] & $\checkmark$ & & \\
\hline FastTrack [14] & $\checkmark$ & $\checkmark$ & \\
\hline RealSyn [10] & & $\checkmark$ & \\
\hline Factest [11] & $\checkmark$ & & \\
\hline Model mismatch (SOS) [35] & $\checkmark$ & & \\
\hline RTD [19] & $\checkmark$ & & \\
\hline Fly-by-Logic [28] & $\checkmark$ & & $\checkmark$ \\
\hline
\end{tabular}

trajectory by maximizing robustness of the STL specification, and then synthesize tracking controllers. Their method can only handle specifications with bounded horizon and does not provide any guarantee against disturbances.

Xiao et al. [41] propose a method for synthesis of distributed controllers for a set of autonomous vehicles in a lane merging situation. They consider only linear systems as vehicle models, use global optimal control to find a nominal controller, and employ local control barrier functions with safety constraints. Their designed controllers is not provably safe in the presence of disturbance and can occasionally violate the safety constraints. Nikou et al. [26] have studied the problem of robust navigation for multi-agent systems based on nominal reference trajectory and pre-computed feedback controllers. Their approach requires sensing capabilities of the agents to avoid collision. In contrast, our method does not requires any sensing capabilities of the agents. Sun et al. [37] have studied motion planning of multi-agent systems with linear temporal logic (LTL) specifications, under the presence of disturbances and denial of service attacks. Their approach uses SMC programming to compute a feasible nominal trajectory and employs feedback controllers to gain robustness. Despite being able to provide guarantees against disturbances, their implementation is centralized, thus the required time increases significantly for high-dimensional reach-avoid specifications.

There are other works that use a pre-defined motion primitive library to perform planning for multi-robot systems [5, 9, 12, 33]. In contrast, our method deals with the dynamical model directly. 
Our construction can also be seen as an assume-guarantee technique that decomposes the global problem based on nominal trajectory tubes. Similar decompositions have been studied in the discrete case $[2,22]$. The closest related work that matches our level of generality is the work by Bansal et al. [4]. However, they assume that each robot has its own reach-avoid specification while avoiding collision with the other robots. In contrast, we allow global reachavoid specifications, which subsume their class of specifications. In fact, there are control problems that can be easily handled by our approach and cannot be encoded in their setting. An example is robots maintaining a formation while fulfilling their tasks [1].

A subset of approaches listed above have available implementations. In Table 1, we summarize the main features of the publicly available tools. We highlight that our tool GAMARA is the only one that fulfills all the criteria.

\section{SYSTEMS AND CONTROLLERS}

Notation. We denote the set of natural numbers including zero by $\mathbb{N}$. We use the notation $\mathbb{R}$ and $\mathbb{R}_{>0}$ to denote respectively the set of real numbers and the set of positive real numbers. We use superscript $n>0$ with $\mathbb{R}$ and $\mathbb{R}_{>0}$ to denote the Cartesian product of $n$ copies of $\mathbb{R}$ and $\mathbb{R}_{>0}$ respectively. Given two points $x=\left(x_{1}, \ldots, x_{n}\right)$ and $y=\left(y_{1}, \ldots, y_{n}\right)$ in $\mathbb{R}^{n}$ and a relational symbol $\triangleright \in\{\leq,<,=,>, \geq\}$, we write $x \triangleright y$ if $x_{i} \triangleright y_{i}$ for every $i \in\{1,2, \ldots, n\}$. The operator $|\cdot|$ is used to denote both the absolute value of a vector and cardinality of a set, depending on the type of the operand, and the operator $\|\cdot\|$ is used to denote the infinity norm.

Let $f: A \rightarrow B$ and $g: C \rightarrow D$ be two functions. We define the product function $f \otimes g: A \times C \rightarrow B \times D, f \otimes g:(a, c) \mapsto(f(a), g(c))$. The product is associative and extends to more than two functions in the obvious way. Given $c \in \mathbb{R}^{n}$ and $\varepsilon \in \mathbb{R}_{>0}^{n}$, the ball with center $c$ and radius $\varepsilon$ in $\mathbb{R}^{n}$ is denoted by $\Omega_{\varepsilon}(c):=\left\{x \in \mathbb{R}^{n} \mid\|x-c\| \leq \varepsilon\right\}$.

Let $A$ be a set. We use the notation $A^{\infty}$ to denote the set of all finite and infinite sequences formed using the members of $A$. Our control tasks are defined using a subset of Linear Temporal Logic (LTL). In particular, we use the until operator $\mathcal{U}$ and the next operator $\bigcirc$ defined as follows. Let $p$ and $q$ be subsets of $\mathbb{R}^{n}$ and $\rho=\left(x_{0}, x_{1}, \ldots\right)$ be an infinite sequence of elements from $\mathbb{R}^{n}$. We write $\rho \models \bigcirc^{k} p$ if $x_{k} \in p$. We write $\rho \vDash p \mathcal{U} q$ if there exists $i \in \mathbb{N}$ s.t. $x_{i} \in q$ and $x_{j} \in p$ for all $0 \leq j<i$. For detailed syntax and semantics of LTL, we refer to the book by Baier et al. [3] and references therein.

Control Systems. A control system $\Sigma=\left(X, x_{\mathrm{in}}, U, W, f\right)$ consists of a state space $X \subseteq \mathbb{R}^{n}$, an initial state $x_{\text {in }} \in X$, an input space $U \subseteq \mathbb{R}^{m}$, a compact disturbance set $W \subset \mathbb{R}^{n}$ containing 0 , and a vector field $f: X \times U \rightarrow X$ modeling the nominal dynamics of the system. A trajectory of $\Sigma$ is a finite or infinite sequence $x_{0}, x_{1}, \ldots \in X^{\infty}$ such that $x_{0}=x_{\text {in }}$ and for each $i \geq 0$, there is an $u_{i} \in U$ and $w_{i} \in W$ such that $x_{i+1}=f\left(x_{i}, u_{i}\right)+w_{i}$. A trajectory is nominal if $w_{i}=0$ for all $i \geq 0$. Intuitively, a control system represents a (possibly nonlinear) dynamical system with control inputs form the set $U$ and disturbances from the set $W$.

Product Control Systems. Let $\left\{\Sigma^{i}\right\}_{i \in[1 ; N]}, \quad \Sigma^{i}=$ $\left(X^{i}, x_{\mathrm{in}}^{i}, U^{i}, W^{i}, f^{i}\right)$, for $i \in[1 ; N]$, be a set of $N$ control systems. The product control system of $\left\{\Sigma^{i}\right\}_{i \in[1 ; N]}$ is defined as the control system $\Sigma^{\times}=\left(X^{\times}, x_{\text {in }}^{\times}, U^{\times}, W^{\times}, f^{\times}\right)$, where
$X^{\times}:=X^{1} \times \ldots \times X^{N}, x_{\text {in }}^{\times}:=\left(x_{\text {in }}^{1}, \ldots, x_{\text {in }}^{N}\right), U^{\times}:=U^{1} \times \ldots \times U^{N}$, $W^{\times}:=W^{1} \times \ldots \times W^{N}$, and $f^{\times}:=f^{1} \otimes \ldots \otimes f^{N}$. We use the state projection operator $\operatorname{proj}^{i}: X^{\times} \rightarrow X^{i}$ with $\operatorname{proj}^{i}\left(x^{1}, \ldots, x^{N}\right)=x^{i}$. For brevity, we write $\left\{\Sigma^{i}\right\}$ instead of $\left\{\Sigma^{i}\right\}_{i \in[1 ; N]}$, when the range of $i$ is irrelevant or clear from context.

Sampled-time Systems. We define control systems and their product over discrete time. Such systems can be obtained as time discretizations of continuous-time nonlinear dynamical systems. We sketch the connection. Consider the tuple $\left(X, x_{\mathrm{in}}, U, W, f\right)$ as above and suppose that $f: X \times U \rightarrow X$ is such that $f(\cdot, u)$ is locally Lipschitz for all $u \in U$. Given a time horizon $\tau>0$, an initial state $x_{0}$, and a constant input $u$, define the continuous time trajectory $\zeta_{x_{0}, u}$ of the system on the time interval $[0, \tau]$ as an absolutely continuous function $\zeta_{x_{0}, u}:[0, \tau] \rightarrow X$ such that $\zeta_{x_{0}, u}(0)=x_{0}$, and $\zeta_{x_{0}, u}$ satisfies the differential inclusion $\dot{\zeta}_{x_{0}, u}(t) \in f\left(\zeta_{x_{0}, u}(t), u\right)+W$ for almost all $t \in[0, \tau]$. Given $\tau, x_{0}$, and $u$, we define $\operatorname{Sol}\left(x_{0}, u, \tau\right)$ as the set of all $x \in X$ such that there is a continuous time trajectory $\zeta_{x_{0}, u}$ with $\zeta(\tau)=x$. A sequence $x_{0}, x_{1}, \ldots$ is a time-sampled trajectory if $x_{0}=x_{\text {in }}$ and for each $i \geq 0$, we have $x_{i+1} \in \operatorname{Sol}\left(x_{i}, u_{i}, \tau\right)$ for some $u_{i} \in U$.

Given a continuous-time nonlinear dynamical system with the tuple $\left(X, x_{\mathrm{in}}, U, W, f\right)$ as above and a sampling time $\tau$, there exist techniques to construct a control system $\Sigma=\left(X, x_{\text {in }}, U, W, f_{\tau}\right)$ such that every time-sampled trajectory of the continuous-time system is also a trajectory of $\Sigma$. We omit the details of the construction; see, e.g., [29].

Controllers. An open-loop controller for $\Sigma=\left(X, x_{\mathrm{in}}, U, W, f\right)$ over a time interval $[0 ; T]$ with $T \in \mathbb{N}$ is a function $C:[0 ; T] \rightarrow U$. The open loop is obtained when we connect $C$ with $\Sigma$ serially, denoted by $C \triangleright \Sigma$. The set of trajectories of the open-loop system $C \triangleright \Sigma$ consists of all finite trajectories $x_{0}, x_{1}, \ldots, x_{T}$ such that $x_{0}=x_{\text {in }}$ and $x_{i+1}=f\left(x_{i}, C(i)\right)+w_{i}$ for some $w_{i} \in W$ for all $i \in[0 ; T-1]$.

A feedback controller for $\Sigma$ over a time interval $[0 ; T], T \in \mathbb{N}$, is a function $C: X \times[0 ; T] \rightarrow U$. We denote the feedback composition of $\Sigma$ with $C$ as $C \| \Sigma$. The set of trajectories of the closed-loop system $C \| \sum$ consists of all finite trajectories $x_{0}, x_{1}, \ldots, x_{T}$ such that $x_{0}=x_{\text {in }}$ and for all $i \in[0 ; T-1]$, we have $x_{i+1}=f\left(x_{i}, C\left(x_{i}, i\right)\right)+w_{i}$ for some $w_{i} \in W$. For both open-loop and feedback composition, the nominal trajectories are the ones for which $w_{i}=0$ for all $i \geq 0$.

Now let $\left\{\Sigma^{i}\right\}$ be a set of control systems. We can define global open-loop and feedback controllers by defining the respective controllers on the product system $\Sigma^{\times}$. We can also define local openloop and feedback controllers $C^{i}$ for each $\Sigma^{i}$. In this latter case, the set of trajectories of the system $\left\{C^{i}\right\} \triangleright\left\{\Sigma^{i}\right\}$ (respectively, $\left\{C^{i}\right\} \|$ $\left.\left\{\Sigma^{i}\right\}\right)$ are finite sequences $x_{0}^{\times}, x_{1}^{\times}, \ldots, x_{T}^{\times}$such that $x_{0}^{\times}=x_{\text {in }}^{\times}$and for each $j \in[0 ; T-1]$, we have $\operatorname{proj}^{i}\left(x_{j+1}^{\times}\right)=f^{i}\left(\operatorname{proj}^{i}\left(x_{j}^{\times}\right), C^{i}(j)\right)+$ $w_{j i}\left(\right.$ respectively, $\operatorname{proj}^{i}\left(x_{j+1}^{\times}\right)=f^{i}\left(\operatorname{proj}^{i}\left(x_{j}^{\times}\right), C^{i}\left(\operatorname{proj}^{i}\left(x_{j}^{\times}\right), j\right)\right)+$ $w_{j i}$ ) for some $w_{j i} \in W^{i}$, for each $i \in[1 ; N]$.

Decentralized Controller Synthesis Problem. Let $\left\{\Sigma^{i}\right\}$ be a set of control systems. A (global) control specification $\mathcal{L}$ is a set of finite sequences in $\left(X^{\times}\right)^{*}$. Intuitively, a control specification specifies a set of "good behaviors" of the product system. The decentralized open-loop (resp. feedback) controller synthesis problem asks, given $\left\{\Sigma^{i}\right\}$ and a global control specification $\mathcal{L}$, to construct a set of local open-loop (resp. feedback) controllers $\left\{C^{i}\right\}$ such that $\left\{C^{i}\right\} \triangleright\left\{\Sigma^{i}\right\}$ 
(resp. $\left\{C^{i}\right\} \|\left\{\Sigma^{i}\right\}$ ) realizes the global control specification $\mathcal{L}$, i.e., all trajectories of the respective systems belong to $\mathcal{L}$.

In particular, we consider reach-avoid specifications, written as $\neg$ Avoid $\mathcal{U}$ Goal in linear temporal logic, for two subsets Avoid, Goal $\subseteq X^{\times}$. A trajectory $x_{0}, x_{1}, \ldots$ satisfies the reach avoid specification if there is some $j \in \mathbb{N}$ such that $x_{j} \in$ Goal and for all $i \in[0 ; j-1]$, we have $x_{i} \notin$ Avoid.

Our notion of product of control systems is a Cartesian product of each component. Thus, we assume that the dynamics of individual control systems are not coupled. However, the overall trajectories of the control systems can be coupled through the control objective. Indeed, our choice of the specification $\Phi$ on the product state space $X^{\times}$subsumes many interesting class of control tasks. For example, we can express situations when the robots have their own local reach-avoid specifications, and they need to avoid collision among each other; we consider such a specification in our examples in Sec. 4.1. In addition, we can also specify more general tasks which cannot be easily decomposed into individual subtasks for the robots. One example is the formation control problem, which we consider in the example in Sec. 4.2, where a set of robots need to reach some location while maintaining a given geometric formation. Since the formation is defined using the relative positions of the robots, it is not possible to decompose this task into separate local reach-avoid subtasks.

Example 2.1. Consider two simple one-dimensional control systems $\Sigma^{i}=\left(X^{i}, x_{\mathrm{in}}^{i}, U^{i}, W^{i}, f^{i}\right), i \in\{1,2\}$, whose states evolve over the real line, and at every time step, the values of their states can be either incremented or decremented by 1 independently. We ignore the effect of the disturbances for simplicity. More formally, $X^{1}=X^{2}=\mathbb{R}, U^{1}=U^{2}=\{-1,1\}, W^{1}=W^{2}=\{0\}$, and $f^{1}\left(x^{1}, u^{1}\right) \equiv x^{1}+u^{1}, f^{2}\left(x^{2}, u^{2}\right) \equiv x^{2}+u^{2}$. Suppose the initial states are $x_{\text {in }}^{1}=0$ and $x_{\text {in }}^{2}=5$, and assume that the specification requires them to simultaneously reach the respective goal sets Goal $^{1}=$ $\{10\}$ and Goal $^{2}=\{20\}$ while maintaining a distance of at least 5 among themselves. This specification can be formulated as a reachavoid objective ( $\neg$ Avoid $\mathcal{U}$ Goal) for the overall two-dimensional product system, where Avoid $=\left\{\left(x^{1}, x^{2}\right) \in \mathbb{R}^{2}|| x^{1}-x^{2} \mid<5\right\}$ and Goal $=\{(10,20)\}$. On the other hand, a decomposition of this objective into independent reach-avoid sub-tasks for $\Sigma^{1}$ and $\Sigma^{2}$ is not possible, because the task has been specified in terms of their relative distance and their simultaneous arrival at the goal sets.

\section{MULTI-AGENT REACH-AVOID SYNTHESIS}

\subsection{Problem Statement}

We now consider the decentralized controller synthesis problem for a set of control systems $\left\{\Sigma^{i}\right\}$ w.r.t. a global reach-avoid specification $\Phi=\neg$ Avoid $\mathcal{U}$ Goal, where Avoid, Goal $\subseteq X^{\times}$are subsets of the product state space.

First, we define a robust version of the control specification. Let $\varepsilon \in \mathbb{R}_{>0}^{n}$ be a robustness margin. We define the $\varepsilon$-robust version of $\Phi$, denoted by $\Phi_{\varepsilon}:=\left(\neg\right.$ Avoid $^{\prime} \mathcal{U}$ Goal $\left.^{\prime}\right)$, where Avoid' $=$ Avoid $\oplus \Omega_{\varepsilon}(0)$ and Goal ${ }^{\prime}=$ Goal $\ominus \Omega_{\varepsilon}(0)$, and $\oplus$ and $\ominus$ are set operators denoting the Minkowski addition and difference, respectively. Intuitively, if a trajectory $x_{0}, x_{1}, \ldots$ satisfies $\Phi_{\varepsilon}$, then any trajectory $y_{0}, y_{1}, \ldots$ such that $\left\|x_{i}-y_{i}\right\| \leq \varepsilon$ satisfies $\Phi$.
Problem 1 (Decentralized Controller Synthesis). Inputs: Control systems $\Sigma^{i}=\left(X^{i}, x_{\text {in }}^{i}, U^{i}, W^{i}, f^{i}\right), i \in[1 ; N]$, and global specification $\Phi=\neg$ Avoid $\mathcal{U}$ Goal.

Parameters: A robustness margin $\varepsilon \in \mathbb{R}_{>0}^{n}$.

Outputs: Local feedback controllers $\left\{C^{i}\right\}$ for $\left\{\Sigma^{i}\right\}, i \in[1 ; N]$, such that $\left\{C^{i}\right\} \|\left\{\Sigma^{i}\right\}$ realizes $\Phi$.

It is important to notice that any solution for this problem is required to provide a formal guarantee on the satisfaction of $\Phi$, i.e., the reach-avoid specification $\Phi$ must be satisfied under every value of the disturbances affecting the control systems. Further, the solution must not require any information exchange between the different agents. Embedding this feature simplifies implementation by eliminating the need for regular synchronization between agents at run time.

\subsection{Solution Outline}

To solve the decentralized control problem, we first plan a highlevel nominal trajectory for the product system by ignoring the disturbances, and then synthesize low-level formally verified controllers for robustly tracking the nominal trajectory under worstcase disturbances. We summarize our approach for solving Prob. 1 in Alg. 1. The approach is composed of three main steps: (1) Synthesize a global open-loop controller for the nominal system as a single planner task on the product system to satisfy $\Phi_{\varepsilon}$; (2) Project the controller into local controllers and obtain a nominal trajectory for each system; and (3) Design local closed-loop controllers to track the nominal trajectory while always staying within the robustness margin. The soundness of the technique is summarized below.

THeorem 3.1. Local feedback controllers $\left\{C^{i}\right\}$ synthesized by Alg. 1 guarantee that the the product system $\left\{C^{i}\right\} \|\left\{\Sigma^{i}\right\}$ realizes the global specification $\Phi$.

Proof. Note that $\Phi_{\varepsilon}$ is a stronger version of $\Phi$ and is intentionally made conservative to allow for $\varepsilon$-deviation in the trajectory of the product system. Since $\left\{\rho^{i}\right\}$ is the unique solution of the nominal product system and satisfies $\Phi_{\varepsilon}$, it is guaranteed that $\varepsilon^{-}$ perturbation of this nominal trajectory satisfies $\Phi$. It can be observed that all solutions of $\Sigma^{i}$ stay within distance $\varepsilon^{i}$ of the nominal trajectory $\rho^{i}$ regardless of the disturbance. This completes the proof.

Next, we discuss some implementation details of Alg. 1 for the global open-loop planner (Step 1) and the local guaranteed trajectory tracking (Step 3) in our tool GAMARA. Note that Step 2 is a simple projection from the product space into local spaces. While we instantiate particular techniques, our method can be used with other implementations as well.

\subsection{Open-loop Planning}

The planner used for generating nominal trajectories in Step 1 of our algorithm should be fast and scalable. In addition, it should be capable of handling non-linear dynamics and constraints. Our choice for the planner is ALTRO [15]. ALTRO is a fast and numerically robust solver for constrained trajectory optimization problems and and is capable of handling nonlinear state and input constraints. Given a product system $\Sigma_{n o m}^{\times}$, reach-avoid specification 


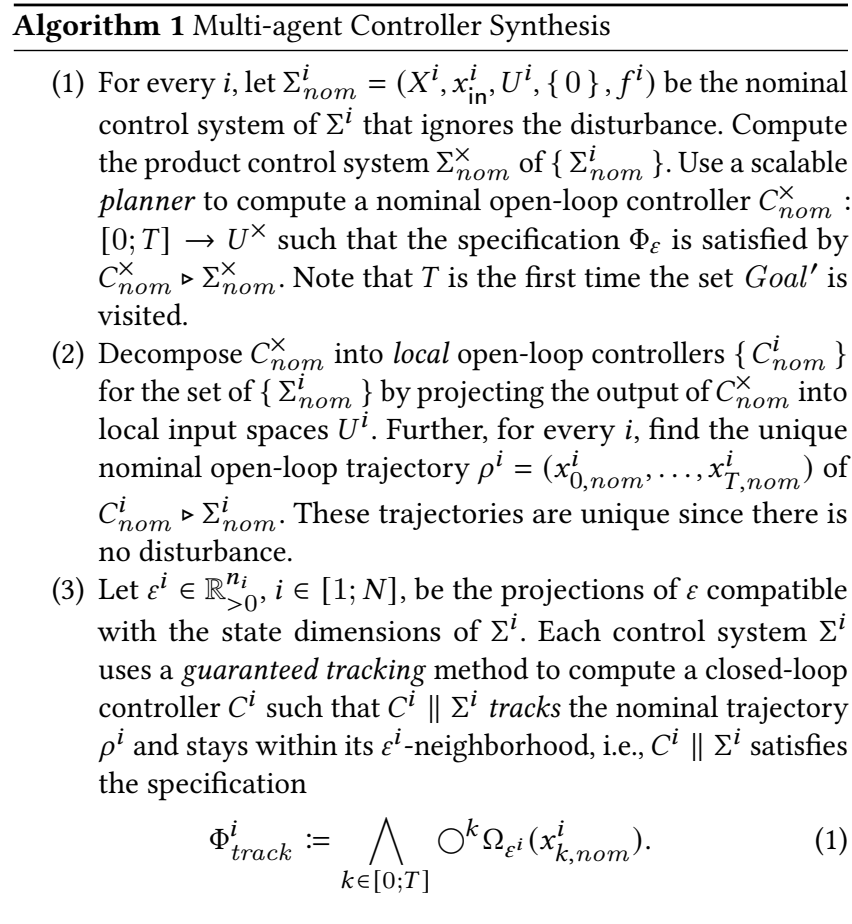

$\Phi_{\varepsilon}$ and time horizon $T$, ALTRO computes an open-loop controller $C_{\text {nom }}^{\times}:[0 ; T] \rightarrow U^{\times}$by solving the optimization

$$
\begin{array}{ll}
\underset{u_{0}^{\times}, u_{1}^{\times}, \ldots, u_{T}^{\times}}{\operatorname{minimize}} & \ell_{T}\left(x_{T}^{\times}\right)+\sum_{k=0}^{T-1} \ell_{k}\left(x_{k}^{\times}, u_{k}^{\times}\right) \\
\text {subject to } & x_{k+1}^{\times}=f^{\times}\left(x_{k}^{\times}, u_{k}^{\times}\right), \quad \forall k \in[0 ; T-1] \\
& g\left(x_{k}^{\times}, u_{k}^{\times}\right) \leq 0, \quad \forall k \in[0 ; T] \\
& h\left(x_{k}^{\times}, u_{k}^{\times}\right)=0, \quad \forall k \in[0 ; T],
\end{array}
$$

where $\ell_{k}(\cdot, \cdot)$ denotes a quadratic objective function assigning cost to each pair of state and input before the end of horizon, $\ell_{T}(\cdot)$ represents a quadratic objective function assigning penalty to the final state $x_{T}^{\times}$being away from the goal set Goal'. The constraints $g\left(x_{k}^{\times}, u_{k}^{\times}\right) \leq 0$ and $h\left(x_{k}^{\times}, u_{k}^{\times}\right)=0$ capture the requirement that at each time $k$ the state should not be in Avoid', the state $x_{T}^{\times}$should be in $G o a l^{\prime}$, and the input $u_{k}^{\times}$should always be in $U^{\times}$. In multirobot scenarios, the inequality constraints can be used to define collision and obstacle avoidance specifications and the equality constraints can define fixed formation specification. Note that the reach-avoid specification is fulfilled if the corresponding equality and inequality constraints (i.e., $g(\cdot) \leq 0, h(\cdot)=0$ ) are satisfied at every time-step and thus choice of the quadratic objective function ( $\ell_{k}$ for $\left.k \in[0 ; T]\right)$ is not crucial.

REMARK 1. ALTRO only supports bounded horizon control problems. For this reason, we model the states in Goal' as a sink state and select a time horizon $T$ for solving the planning task on the nominal product system. We increase the horizon $T$ if ALTRO is not able to find a controller. We remark that this is an ALTRO-specific implementation detail, and our overall method does not rely on a fixed time horizon.

\subsection{Guaranteed Trajectory Tracking}

Trajectories computed in the planning stage might not be followed in the presence of disturbance and therefore we need to use a formally guaranteed tracking controller to satisfy the given reachavoid specification. We use abstraction-based controller design $(A B C D)$ for Step 3. ABCD can handle nonlinear dynamics, (bounded) uncertainties, and $\omega$-regular specifications. In particular, we use the implementation of ABCD in the tool called SCOTS [31]. Next, we introduce briefly the basics of $\mathrm{ABCD}$. For simpler notation, we omit the control system index $i$ in the rest of this section.

Finite-state Abstraction of Control Systems. Let $\Sigma=$ $\left(X, x_{\text {in }}, U, W, f\right)$ be a control system and Domain be a subset of $X$ which imposes a safety specification on all possible trajectories of the system. Let $\widehat{X}$ be a given finite partition of Domain, and $\widehat{U}$ be a finite subset of equally spaced (w.r.t. infinity norm on $\mathbb{R}^{m}$ ) points in the input set $U$. A finite-state abstraction of $\Sigma$ is a finite state-transition system $(\widehat{X}, \widehat{U}, \widehat{f})$, where $\widehat{x}^{\prime}$ is in $\widehat{f}(\widehat{x}, u)$ if there is a pair of states $x \in \widehat{x}$ and $x^{\prime} \in \widehat{x}^{\prime}$ such that $x^{\prime} \in \operatorname{Sol}_{\Sigma}(x, u)$.

In this work, we will use uniformly sized rectangular partition elements to construct the set $\widehat{X}$ from the set Domain. Without going into the detail of the construction, we assume that the size of the partition elements is provided as a vector $\eta_{x} \in \mathbb{R}_{>0}^{n}$ which is an input to the abstraction procedure. Note that, the larger $\eta_{x}$ is (where comparison is made dimension-wise), the smaller is the state space $\widehat{X}$ resulting in an efficient computation. On the other hand, the smaller $\eta_{x}$ is, the better is the precision of the abstraction $\widehat{\Sigma}$ increasing the chance of a successful controller synthesis. Similar to the state space partition size $\eta_{x}$, we also assume that the set $\widehat{U}$ is chosen based on an input space discretization parameter $\eta_{u} \in \mathbb{R}_{>0}^{m}$ that governs the distance between the points in $\widehat{U}$.

Feedback Refinement Relation. Let $\Sigma$ be a control system and $\widehat{\Sigma}$ be its finite-state abstraction. A feedback refinement relation (FRR) from $\Sigma$ to $\widehat{\Sigma}$ is a relation $Q \subseteq$ Domain $\times \widehat{X}$ s.t. for all $x \in$ Domain there is some $\widehat{x} \in \widehat{X}$ such that $Q(x, \widehat{x})$ and for all $(x, \widehat{x}) \in Q$, we have (i) $\widehat{U}_{\widehat{\Sigma}}(\widehat{x}) \subseteq U_{\Sigma}(x)$, and (ii) $u \in U_{\widehat{\Sigma}}(\widehat{x}) \Rightarrow$ $Q(f(x, u)) \subseteq \widehat{f}(\widehat{x}, u)$, where $U_{\Sigma}(x):=\{u \in U \mid f(x, u) \neq \emptyset\}$ and $\widehat{U}_{\widehat{\Sigma}}(\widehat{x}):=\{u \in \widehat{U} \mid \widehat{f}(\widehat{x}, u) \neq \emptyset\}$. We write $\Sigma \varsigma_{Q} \widehat{\Sigma}$ if $Q$ is an FRR from $\sum$ to $\widehat{\Sigma}$.

Abstraction-Based Controller Design. The abstraction-based controller design (ABCD) [29] is a 3-step method to find a robust controller for the control system $\Sigma$ : First, we compute a finite state abstraction $\widehat{\Sigma}$ s.t. $\Sigma \leq_{Q} \widehat{\Sigma}$. Second, we synthesize an abstract controller of the form $\widehat{C}: \widehat{X} \rightarrow \widehat{U}$ for $\widehat{\Sigma}$ using methods from the reactive synthesis literature. Finally, we obtain the desired controller $C$ as $C:=\widehat{C} \circ Q$. It is known that this three step process produces a controller $C$ such that $C \| \Sigma$ satisfies the specification [29].

If a controller cannot be found, we reduce the discretization parameters $\eta_{x}$ and $\eta_{u}$ and try again, or use a larger robustness margin $\varepsilon$.

Optmization: Local $A B C D$ around the nominal trajectory. The abstraction process of $\mathrm{ABCD}$ usually requires computation of abstract transitions over the whole compact set Domain, which is computationally expensive. Luckily, for Step 3 of Alg. 1, we only need to compute transitions in the $\varepsilon$-neighborhood of the given 
nominal trajectory. Given a control system $\Sigma$, together with a reference open-loop trajectory $\rho=\left(x_{0, n o m}, \ldots, x_{T, n o m}\right)$ and a tube size $\varepsilon \in \mathbb{R}_{>0}^{n}$, we iteratively construct a tube as union of $\varepsilon$-balls around the reference trajectory (note that we have omitted the system index $i$ for simpler notation). Next, we compute finite state abstraction for $\Sigma$ for the chosen parameters $\eta_{x}, \eta_{u}$ by setting

$$
\text { Domain }:=\bigcup_{k=0}^{T} \Omega_{\varepsilon}\left(x_{k, n o m}\right) \text {. }
$$

In practice, this local computation of the abstraction is the key to scalability.

REMARK 2. Our decentralized controller synthesis approach features an interplay between the global open-loop planning and local formal synthesis via the robustness parameter $\varepsilon$ and the discretization parameters $\eta_{x}, \eta_{u}$. The parameter $\varepsilon$ should be large enough to allow deviation from the nominal trajectory caused by the disturbance. A small \& makes the local specification $\Phi_{\text {track }}^{i}$ difficult for ABCD synthesis thus requiring large computational complexity with smaller discretization parameters $\eta_{x}, \eta_{u}$. On the other hand, large $\varepsilon$ makes the specification $\Phi_{\varepsilon}$ very conservative or infeasible for the global openloop planning. Therefore, appropriate parameters should be selected iteratively for a successful controller synthesis.

\subsection{Hybrid vs Geometric Planning}

Note that Step 3 of Alg. 1 does not use the nominal controller obtained in Step 1 and requires only the nominal trajectories. Then one could argue that, instead of using ALTRO to generate nominal trajectories, a fast geometric planner [17] can be employed to generate geometric plans. However, fast geometric planners do not usually take into consideration the dynamics and control constraints. In our experience, the plans for nominal trajectories generated while ignoring the system dynamics are often untrackable unless the underlying system has special properties (e.g., differential flatness [25]). This is especially true for systems with restricted control capabilities or under-actuated systems. We demonstrate this phenomenon on a control system $\Sigma$ that is a simple 2-dimensional pendulum with the following nominal dynamics:

$$
\dot{x}_{1}=x_{2} \quad \dot{x}_{2}=-\sin \left(x_{1}\right)+u / 5,
$$

where $x_{1}$ represents the angle (in Radian) of the pendulum rod measured counterclockwise from the vertical downward position, and $x_{2}$ represents the rate of change of $x_{1}$ or the angular velocity. Suppose the initial state of the pendulum is $(0,0)$, i.e., when

Figure 3: The state trajectory of the inverted pendulum: the red point is the initial state, the green point is the final state, the blue spiral is the nominal trajectory obtained from ALTRO, and the dashed straight line is a geometric plan. the pendulum is in the vertical downward position and is stationary. Suppose we want to find a controller for the goal Goal $=\{(\pi, 0)\}$, i.e., when the pendulum is in the vertical upward position and is stationary. The set of unsafe states Avoid is empty, i.e., no safety constraint is imposed. When we use ALTRO to compute an open-loop controller $C$, unsurprisingly, the controlled trajectory of $C \triangleright \sum$ looks like a spiral, as shown in blue Fig. 3 .

The synthesis of tracking controller using $\mathrm{ABCD}$ is indeed successful when we feed this nominal trajectory to our $\mathrm{ABCD}$ solver. However, if we use a geometric planner for this example that ignores the dynamics, the nominal trajectory would be a straight line path from $(0,0)$ to $(\pi, 0)$ (shown using a dashed line in Fig. 3), which gives an infeasible tracking problem for $\mathrm{ABCD}$, due to the restrictions on possible trajectories of the pendulum coming from the its dynamics.

\section{EXPERIMENTAL EVALUATION}

We have implemented our approach (as presented in Sec. 3) in the open source tool GAMARA. ${ }^{1}$ We evaluate the effectiveness of GAMARA on two distinct categories of problems: local reach-avoid problems with collision avoidance and global formation control problems. We consider four case studies: multi-drone path planning, crane and vehicle, lane merging, and multi-drone formation control. The design of nominal controller using ALTRO for all experiments was performed on a machine with core i5-4590 CPU at $3.30 \mathrm{GHz}$, with 16 GB of RAM. The formal controller synthesis using SCOTS for all systems except crane system was performed on the same machine. Controller synthesis for crane system is done on a cluster with 4 Intel Xeon E7-8857 v2 CPUs (48 cores in total) at $3 \mathrm{GHz}$, with $1.5 \mathrm{~TB}$ of RAM.

In all of our case studies, the robots are moving in a twodimensional shared workspace (related but not exactly the same as the robots' state spaces) that possibly has obstacles. Table 2 shows run times for different stages of each experiment. For local $A B C D$, the reported numbers correspond to the maximum value among all of the agents. This choice is due to the fact that feedback controllers for different agents can be computed independently in parallel over different machines. To provide a more fine-grained comparison, Fig. 4 shows the variations of run times of the different local ABCD tasks for every experiment. We have excluded the crane and vehicle case study in the figure due to an expected large variance originating from different dynamics. Notice that a higher number of state-input pairs does not necessarily result in a higher run time for local $\mathrm{ABCD}$ as the number of transitions and features of the parallel implementation can play a role. We compare GAMARA with ABCD applied to the product system to satisfy the global specification. As reflected in Table 2, memory requirement for global ABCD exceeds both system's (laptop and cluster) limit (1.5TB of RAM) in all of the experiments.

\subsection{Local reach-avoid with collision avoidance}

We first consider situations when each robot has an individual reachability specification, and they need to ensure a minimum safe distance from each other and the obstacles.

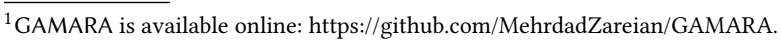


Table 2: Run times for four case studies. Run times (in seconds) for computing open-loop controllers over the corresponding product spaces using ALTRO $\left(T_{g}^{\text {plan }}\right)$, number of state-input pairs of the finite abstraction for the largest ABCD task $\left(N_{l}\right)$, abstraction and synthesis times in SCOTS for that task (respectively $T_{l}^{a b s}$ and $T_{l}^{s y n}$ ), number of state-input pairs of the finite state abstraction for global ABCD $\left(N_{g}\right)$, abstraction and synthesis times for computing a global controller for the product system using SCOTS (respectively $T_{g}^{a b s}$ and $T_{g}^{s y n}$ ). "OOM" denotes "out of memory" on a 1.5TB RAM machine.

\begin{tabular}{|l|c|rrr|rcc|}
\hline Case-study & Global planning & \multicolumn{3}{|c|}{ Local ABCD } & \multicolumn{3}{c|}{ Global ABCD } \\
& $T_{g}^{\text {plan }}$ & $N_{l}$ & $T_{l}^{\text {abs }}$ & $T_{l}^{\text {syn }}$ & $N_{g}$ & $T_{g}^{\text {abs }}$ & $T_{g}^{\text {syn }}$ \\
\hline Multi-drone path planning & 77.85 & $1.13 \times 10^{8}$ & 30.75 & 6.66 & $2.70 \times 10^{110}$ & OOM & OOM \\
\hline Crane and vehicle & 0.65 & $8.56 \times 10^{8}$ & 511.24 & 91.43 & $2.16 \times 10^{18}$ & OOM & OOM \\
\hline Lane merging & 89.02 & $1.07 \times 10^{8}$ & 22.79 & 5.29 & $1.69 \times 10^{59}$ & OOM & OOM \\
\hline Multi-drone formation control & 114.34 & $1.55 \times 10^{8}$ & 39.46 & 7.83 & $3.65 \times 10^{50}$ & OOM & OOM \\
\hline
\end{tabular}

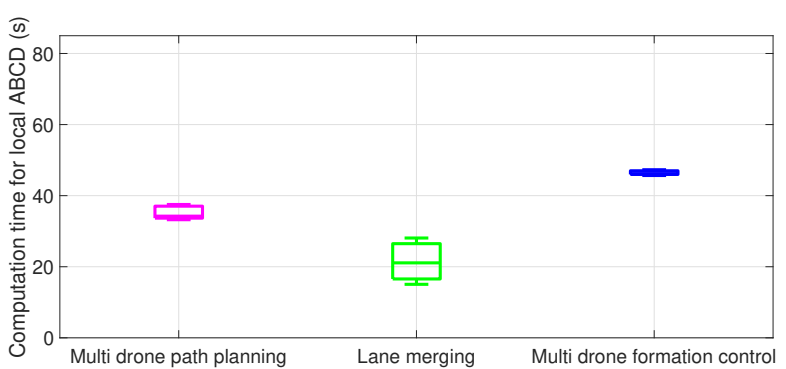

Figure 4: Variations of run times of local ABCD among different agents for three case studies.

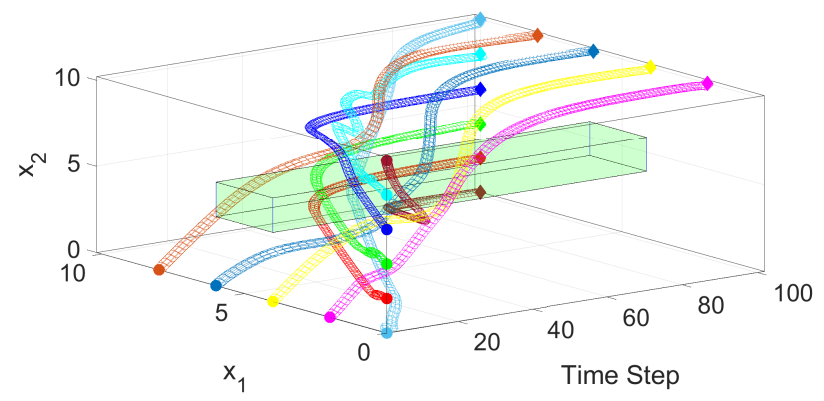

Figure 5: Time-state space illustration of tubes enclosing nominal trajectories for the multi-drone path planning

Suppose $\left\{\Sigma^{i}\right\}$ models a set of robots, Goal ${ }^{i} \subseteq X^{i}$ are the individual goal sets, and $\delta \in \mathbb{R}_{>0}$ is a safety margin for collisions. We consider each robot as a point object with a bounding box for its physical dimensions. The parameter $\delta$ is chosen to be a constant greater than twice the radius of the bounding box around each robot. By keeping a distance at least $\delta$ from the other robots and the obstacles, the robots can avoid collision in their physical domain. The parameter $\delta$ can additionally take into account statutory minimum safe distances among the robots, such as in autonomous driving-like scenarios. The choice of $\delta$ is completely independent of the choice of $\varepsilon$. The latter is a robustness margin introduced to take into account the deviation of the system trajectories under external disturbances. Suppose the specification requires that each robot $\Sigma^{i}$ eventually reaches Goal ${ }^{i}$ while avoiding the obstacle $O b s \subseteq \mathbb{R}^{2}$ and collision with robots by the margin $\delta$. The global specification on the product system $\Sigma^{\times}$is as follows:

- The goal set $G o a l \subseteq X^{\times}$is defined as Goal := Goal ${ }^{1} \times \ldots \times$ Goal $^{N} \subseteq X^{\times}$, and

- The avoid set Avoid $\subseteq X^{\times}$is defined as

$$
\left\{\begin{array}{c|c}
x^{\times} \in X^{\times} & \begin{array}{c}
\exists i \in[1 ; N] \cdot d_{O b s}\left(x^{i}, O b s\right) \leq \delta \\
\vee \\
\exists i, j \in[1 ; N] \cdot i \neq j \cdot d_{C o l}\left(x^{i}, x^{j}\right) \leq \delta
\end{array}
\end{array}\right\},
$$

where $x^{i}$ denotes the component of $x^{\times}$corresponding to $\Sigma^{i}, d_{C o l}(\cdot, \cdot)$ denotes a distance metric for measuring the geometric distance between positions of two systems located in two-dimensional space, and $d_{O b s}(\cdot, \cdot)$ denotes a distance metric for measuring the geometric distance between the position of one system and the obstacle $O b s \subseteq \mathbb{R}^{2}$.

In this category of problems, we apply our approach to three case studies as briefly discussed next. The detailed models for the systems and their different parameters have been presented in Sec. A.1-A.3 in the appendix, and the performance of GAMARA for these experiments have been summarized in the first three rows on Table 2.

4.1.1 Multi-drone path planning. We consider a planning scenario for ten identical drones $(N=10)$. The control objective is to synthesize a feedback controller for each drone so that in the presence of (bounded) disturbance, beginning from the specified initial state, the corresponding target state is reached within a finite horizon, while avoiding collision with other drones and the physical obstacle at every time point. Fig. 5 gives a time-space illustration for the safe tubes around the nominal trajectories. The tracking feedback controllers are synthesized such that every drone remains within its safe tube until reaching its destination, even with worst-case disturbance. Additional analysis and detailed models for the systems and their different parameters are presented in Sec. A.1 in the appendix.

4.1.2 Crane and Vehicle. The goal of this example is to study the performance of our method for controlling a number of robots with different dynamics. As described in Sec. 1, the goal is to move the overhead crane and the vehicle such that they do not collide. Formally guaranteed controllers are computed such that the generated open loop trajectory (see Fig. 2 (left)) is tracked even under 


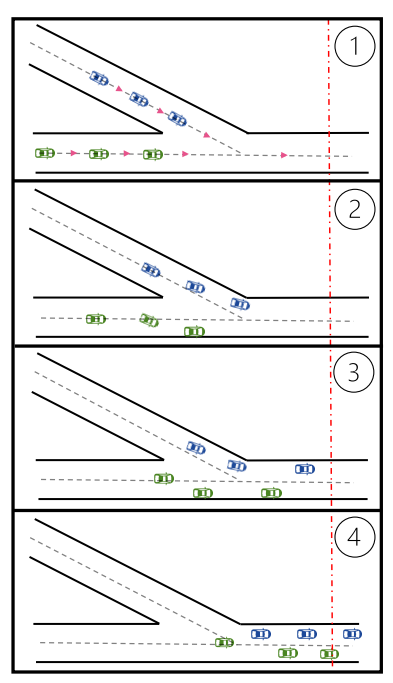

Figure 6: Illustration of a sample trajectory generated by formally guaranteed controllers for the lane merging example

disturbance. More detailed discussion on dynamics of systems and further analysis are presented in Sec. A.2 in the appendix.

4.1.3 Lane Merging. We study a lane merging problem wherein multiple controlled vehicles $(N=6)$ are driving over two merging lanes (Fig. 6, top frame). A dangerous situation may occur at the merging point of the two lanes if vehicles are not controlled properly. Different variants of this problem have been studied in the literature (see, e.g., [40, 41]). Without seeking to optimize fuel consumption or travel time, we set the goal to control the vehicles to pass the merging zone safely. In particular, consider a situation where initially three cars are driving on each of the two lanes (Fig. 6, (top)). The control objective for each vehicle is to pass the red dashed line within a finite horizon without hitting the road's sides or colliding with other vehicles. Fig. 6 demonstrates snapshots of one sample trajectory when feedback controllers are employed under the presence of bounded disturbance. Additional analysis, systems' dynamics and parameters are reported in Sec. A.3 in the appendix.

\subsection{Global formation control problem}

The second category of examples are about maintaining a global formation while satisfying a set of reach-avoid specifications. We show how the formation control problem can be expressed using a static obstacle Avoid on the product state space $X^{\times}$.

Let us first formalize the notion of formation. Let $\left\{\Sigma^{i}=\left(X^{i}, x_{\mathrm{in}}^{i}, U^{i}, W^{i}, f^{i}\right)\right\}$ be a set of robots. A formation constraint is a set $\left\{\lambda^{i, j} \in \mathbb{R}\right\}_{i, j \in[1 ; N]}$ where every $\lambda^{i, j}$ specifies the relative Euclidean distance between the projections of state of robot $\Sigma^{i}$ and robot $\Sigma^{j}$.

Now suppose Goal $^{i} \subseteq X^{i}$ are the individual goal states, Obs $\subset$ $\mathbb{R}^{2}$ is a common obstacle $\delta \in \mathbb{R}_{>0}$ is a safety margin, and $\mu \in \mathbb{R}_{>0}$ is a tolerance margin for the formation constraint. The formation control problem then asks to generate controllers $\left\{C_{i}\right\}$ such that every robot $\Sigma^{i}$ eventually reaches Goal $^{i}$ while avoiding $O b s$ by the

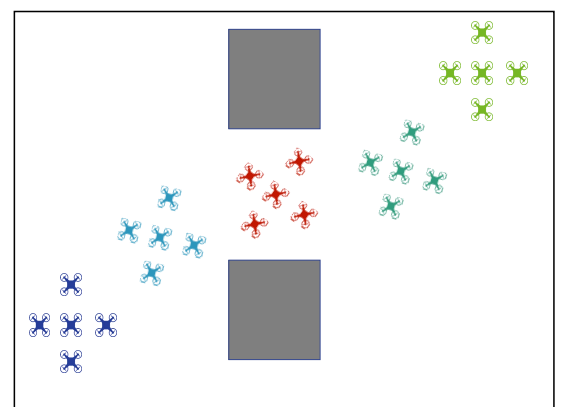

Figure 7: Illustration of a sample trajectory generated by the feedback controllers for the formation control example

margin $\delta$, as well as while making sure that the Euclidean distance between robots $\Sigma^{i}$ and $\Sigma^{j}$ is in the range $\lambda^{i, j} \pm \mu$. Essentially the tolerance margin $\mu$ is to account for the possible slight deviations due to disturbances experienced by the robots. Notice that since the robots have their own goals but at the same time they need to "stay close" to their neighboring robots in the formation for the entire period, they might first need to accompany the other robots to their goals, before being accompanied by them to reach their own goal. We can express the formation control problem in the product state space as follows:

- The goal set $G o a l \subseteq X^{\times}$is defined as Goal := Goal ${ }^{1} \times \ldots \times$ Goal $^{N}$, and

- The avoid set Avoid $\subseteq X^{\times}$is

$$
\left\{\begin{array}{c|c}
x^{\times} \in X^{\times} & \begin{array}{c}
\exists i \in[1 ; N] . D\left(x^{i}, O b s\right) \leq \delta \\
\vee \\
\exists i, j \in[1 ; N] . i \neq j . d\left(x^{i}, x^{j}\right) \notin \lambda^{i, j} \pm \mu
\end{array}
\end{array}\right\},
$$

where the last disjunction in the definition of Avoid is the restriction required for maintaining the formation, and the rest are same as in Sec. 4.1.

4.2.1 Multi-drone formation control. Consider a formation control scenario where a set of five drones (identically modeled) need to go from a specified start point to a certain destination (both defined over the corresponding state spaces) within a finite horizon, while four of them forming a diamond around a fifth drone (positioned at the diamond's center) at every time point. There are two square obstacles from which the group needs to keep a certain minimum distance at all of the time points. Fig. 7 illustrates four sequential frames of a sample perturbed trajectory generated by employing formally guaranteed feedback controllers. Notice that both relative position and orientation between drones are kept (almost) constant throughout the journey. Further analysis can be found in Sec. A.4 in the appendix.

\section{DISCUSSION AND FUTURE WORK}

We have presented a method for synthesizing provably correct decentralized controllers for multi-agent systems with global reachavoid specifications. Our approach is hierarchical. At the top level, we obtain a high-level joint plan by solving a centralized synthesis problem on a simplified model of the product system, and at the bottom level we synthesize local controllers for robustly tracking 
the high-level plan for each individual agent. In this section, we discuss different features of our work, limitations, and future work.

Scalability of the proposed method. We compute the reference trajectory for the product system using ALTRO. Our experiments show that ALTRO generates reference trajectories for highdimension systems quite fast. On the other hand, for tracking controllers, our method synthesizes controllers in a decentralized manner and thus scalability is not affected by increasing the number of agents. We use SCOTS for computing tracking controllers because the algorithm can be effectively parallelized [18]. While GAMARA uses ALTRO and SCOTS for solving the given reach-avoid problem, one could replace them with any other off-the-shelf method that can perform similar tasks.

Extension to richer classes of specifications. Our proposed method is specific to reach-avoid specifications but it can be extended to other specifications by decomposing them into a sequence of reach-avoid tasks. To that end, one can use high-level languages (e.g., $[13,23])$ for specifying complex reach-avoid sequences and invoke GAMARA to solve each individual reach-avoid task.

Choice of parameters. Our algorithm takes as input the robustness margin $\varepsilon$ and the abstraction parameters $\eta_{x}$ and $\eta_{u}$. The larger the value of $\varepsilon$, more difficult it is to synthesize a nominal controller for the product system using ALTRO. On the other hand, the smaller the value of $\varepsilon$, the more difficult it is to synthesize a set of decentralized controllers using SCOTS. Also, there is a trade-off between computation time and ease of synthesis when it comes to choosing the parameters $\eta_{x}$ and $\eta_{u}$ for SCOTS: Larger values of these parameters will result in faster computation but there may be no solution to the synthesis problem. It is not always easy to come up with a good set of parameters. Our current approach adds an outer loop around our tool to search for suitable parameters until the decentralized synthesis is successful. We do not know a good heuristic to systematically explore the parameter space by analyzing the dynamics of the systems.

\section{ACKNOWLEDGMENTS}

This research was sponsored in part by the Deutsche Forschungsgemeinschaft project 389792660 TRR 248-CPEC and by the European Research Council under the Grant Agreement 610150 (ERC Synergy Grant ImPACT)

\section{REFERENCES}

[1] J. Alonso-Mora, E. Montijano, T. Nägeli, O. Hilliges, M. Schwager, and D. Rus Distributed multi-robot formation control in dynamic environments. Autonomous Robots, 43(5):1079-1100, 2019.

[2] R. Alur, S. Moarref, and U. Topcu. Pattern-based refinement of assume-guarantee specifications in reactive synthesis. In TACAS. Springer, 2015.

[3] C. Baier and J.-P. Katoen. Principles of model checking. MIT press, 2008.

[4] S. Bansal, M. Chen, J. F. Fisac, and C. J. Tomlin. Safe sequential path planning of multi-vehicle systems under presence of disturbances and imperfect information In ACC, 2017.

[5] G.B. Banusic, R. Majumdar, M. Pirron, A.K. Schmuck, and D. Zufferey. PGCD robot programming and verification with geometry, concurrency, and dynamics. In ICCPS 2019, Montreal, QC, Canada, 2019.

[6] A.G. Barto, R.S. Sutton, and C.W. Anderson. Neuronlike adaptive elements that can solve difficult learning control problems. IEEE TSMCS, SMC-13(5):834-846, September 1983.

[7] J. Chen, S. Moarref, and H. Kress-Gazit. Verifiable control of robotic swarm from high-level specifications. In AAMAS, pages 568-576. ACM, 2018.
[8] H.M. Choset, S. Hutchinson, K.M. Lynch, G. Kantor, W. Burgard, L.E. Kavraki, and S. Thrun. Principles of robot motion: theory, algorithms, and implementation. MIT press, 2005.

[9] A. Desai, I. Saha, J. Yang, S. Qadeer, and S.A. Seshia. DRONA: A framework for safe distributed mobile robotics. In ICCPS, 2017.

[10] C. Fan, U. Mathur, S. Mitra, and M. Viswanathan. Controller synthesis made real: reach-avoid specifications and linear dynamics. In $C A V$, pages 347-366, 2018.

[11] C. Fan, K. Miller, and S. Mitra. Fast and guaranteed safe controller synthesis for nonlinear vehicle models. In $C A V$, pages 629-652, 2020.

[12] I. Gavran, R. Majumdar, and I. Saha. Antlab: A multi-robot task server. ACM TECS, 16(5s):1-19, October 2017

[13] R. Ghosh, Ch. Hsieh, S. Misailovic, and S. Mitra. Koord: a language for programming and verifying distributed robotics application. OOPSLA, 2020.

[14] S.L. Herbert, M. Chen, S. Han, S. Bansal, J.F. Fisac, and C.J. Tomlin. FaSTrack: A modular framework for fast and guaranteed safe motion planning. In $C D C, 2017$.

[15] T.A. Howell, B.E. Jackson, and Z. Manchester. ALTRO: A fast solver for constrained trajectory optimization. In IROS, 2019.

[16] B.E. Jackson, T.A. Howell, K. Shah, M. Schwager, and Z. Manchester. Scalable cooperative transport of cable-suspended loads with UAVs using distributed trajectory optimization. IEEE Robot. Autom. Lett., 5(2):3368-3374, 2020.

[17] L.E. Kavraki, P. Svestka, J.-C. Latombe, and M.H. Overmars. Probabilistic roadmaps for path planning in high-dimensional configuration spaces. IEEE TRA, 12(4):566-580, 1996.

[18] M. Khaled and M. Zamani. pFaces: an acceleration ecosystem for symbolic control. In HSCC, pages 252-257, 2019

[19] Sh. Kousik, S. Vaskov, Fan Bu, M. Johnson-Roberson, and R. Vasudevan. Bridging the gap between safety and real-time performance in receding-horizon trajectory design for mobile robots. Int. F. Robot. Res., 2020.

[20] S.M. LaValle. Planning Algorithms. Cambridge University Press, 2006.

[21] S.M. LaValle and S.A. Hutchinson. Optimal motion planning for multiple robots having independent goals. IEEE Trans. Robot. Autom., 14(6):912-925, 1998.

[22] R. Majumdar, K. Mallik, A.K. Schmuck, and D. Zufferey. Assume-guarantee distributed synthesis. In EMSOFT, 2020

[23] R. Majumdar, N. Yoshida, and D. Zufferey. Multiparty motion coordination: From choreographies to robotics programs (artifact), 2020.

[24] P.-J Meyer, H. Yin, A.H. Brodtkorb, M. Arcak, and A.J. Sørensen. Continuous and discrete abstractions for planning, applied to ship docking, 2019.

[25] R. M. Murray, M. Rathinam, and W. Sluis. Differential flatness of mechanical control systems: A catalog of prototype systems. In Proceedings of the 1995 ASME International Congress and Exposition, 1995.

[26] A. Nikou and D. V. Dimarogonas. Decentralized tube-based model predictive control of uncertain nonlinear multiagent systems. Int. F. Robust Nonlinear Control, 29(10):2799-2818, March 2019.

[27] P. Nilsson and A.D. Ames. Barrier functions: Bridging the gap between planning from specifications and safety-critical control. In CDC, 2018.

[28] Y.V. Pant, H. Abbas, R.A. Quaye, and R. Mangharam. Fly-by-Logic: Control of multi-drone fleets with temporal logic objectives. In ICCPS, 2018.

[29] G. Reissig, A. Weber, and M. Rungger. Feedback refinement relations for the synthesis of symbolic controllers. IEEE TAC, 62(4):1781-1796, 2016.

[30] A. Rodionova, Y. Pant, K. Jang, H. Abbas, and Rahul Mangharam. Learning-to-Fly: Learning-based collision avoidance for scalable urban air mobility. ArXiv, 2020.

[31] M. Rungger and M. Zamani. SCOTS: A tool for the synthesis of symbolic controllers. In HSCC, 2016.

[32] S.J. Russell and P. Norvig. Artificial Intelligence - A Modern Approach. Pearson Education, 2010.

[33] I. Saha, R. Rattanachai, V. Kumar, G. J. Pappas, and S.A. Seshia. Implan: Scalable incremental motion planning for multi-robot systems. In ICCPS, 2016.

[34] Y.E. Sahin, P. Nilsson, and N. Ozay. Provably-correct coordination of large collections of agents with counting temporal logic constraints. In ICCPS, 2017.

[35] S. Singh, M. Chen, S.L. Herbert, C.J. Tomlin, and M. Pavone. Robust tracking with model mismatch for fast and safe planning: an SOS optimization approach. In WAFR, pages 545-564, 2018.

[36] M. Srinivasan, S. Coogan, and M. Egerstedt. Control of multi-agent systems with finite time control barrier certificates and temporal logic. In CDC, 2018.

[37] X. Sun, R. Nambiar, M. Melhorn, Y. Shoukry, and P. Nuzzo. DoS-resilient multirobot temporal logic motion planning. In ICRA, pages 6051-6057, 2019.

[38] R. Tedrake, I.R. Manchester, M. Tobenkin, and J. W. Roberts. Lqr-trees: Feedback motion planning via sums-of-squares verification. Int. f. Robot. Res, 2010.

[39] T. Wongpiromsarn, U. Topcu, and R.M. Murray. Receding horizon temporal logic planning. IEEE TAC, 57(11):2817-2830, 2012.

[40] W. Xiao and C.G. Cassandras. Decentralized optimal merging control for connected and automated vehicles with optimal dynamic resequencing. In ACC, 2020.

[41] W. Xiao, C.G. Cassandras, and C. Belta. Decentralized merging control in traffic networks with noisy vehicle dynamics: a joint optimal control and barrier function approach. In ITSC, 2019.

[42] L. Yang and N. Ozay. Provably-correct fault tolerant control with delayed information. In $C D C, 2017$. 


\section{A EXAMPLES}

\section{A.1 Multi-drone path planning}

Every drone is modeled as a control system $\Sigma^{i}=\left(X, x_{\mathrm{in}}^{i}, U, W, f_{\tau}^{d}\right)$, where $f_{\tau}^{d}$ is the sampled-time abstraction of the following continuous dynamics:

$$
f^{d}(x(t), u(t)):=\left[\begin{array}{c}
\dot{x}_{1} \\
\dot{x}_{2} \\
\dot{x}_{3}
\end{array}\right]=\left[\begin{array}{c}
u_{1} \cos \left(x_{3}\right) \\
u_{1} \sin \left(x_{3}\right) \\
u_{2}
\end{array}\right] .
$$

where $x_{1}$ and $x_{2}$ denote the drone's position in two-dimensional space, $x_{3}$ denotes the rotational angle, and $u_{1}$ and $u_{2}$ represent control inputs for each drone. Choosing a sampling time $\tau=0.1$ $\mathrm{s}$, the nominal dynamics $f_{\tau}^{d}$ can be characterized uniquely. We consider state and input spaces to be $X=[-1,11]^{2} \times[-2,3.3]$ and $U=[-2.4,2.4]^{2}$, respectively. The disturbance set and robustness margin are chosen as $|W| \leq(0,0.025,0.025)$ and $\varepsilon=$ $(0.20,0.20,0.24)$.

Recall that we consider 10 drones, i.e., $N=10$. Selecting the horizon length $T=104$ and minimum safe distance $\delta=0.24 \mathrm{~m}$, ALTRO computes a valid open-loop trajectory in 77.8 seconds for the product system with 30 state and 20 input variables. Fig. 5 gives time-space illustration for the safe tubes around the nominal trajectories.

For $\mathrm{ABCD}$, we set $\eta_{X}=(0.025,0.025,0.03)$ and $\eta_{U}=(0.3,0.3)$. Tab. 2 shows the run times and number of state-input pairs corresponding to both local and global ABCD. Noticeably, already when $N=2$, memory requirement for global ABCD exceeds memory limits, even 1.5 TB RAM on a cluster machine is not sufficient to synthesize a controller.

On the other hand, using ALTRO alone would not provide guarantee against bounded disturbance. Fig. 9 illustrates the performance of open-loop and feedback controllers in regulating distance between two particular the drones with and without disturbances. As expected, in the absence of disturbances, the openloop controllers suffice and the distance between the two drones (shown in solid blue) does not go below the defined threshold. Next, we consider the case when constant additive disturbances $(0,0.025,0.025)$ and $(0,-0.025,-0.025)$ are being applied to the two drones throughout the whole horizon. It can be noticed that applying the open-loop controller causes that distance between the two drones (shown in solid yellow) to go below the predefined threshold. However, the feedback controller is capable of maintaining distance (shown in solid red) within the safe region when the same disturbance is being applied.

\section{A.2 Crane and Vehicle}

We model the crane and vehicle as control systems $\Sigma^{1}=$ $\left(X^{1}, x_{\mathrm{in}}^{1}, U^{1}, W^{1}, f_{\tau}^{c}\right)$ and $\Sigma^{2}=\left(X^{2}, x_{\mathrm{in}}^{2}, U^{2}, W^{2}, f_{\tau}^{l}\right)$, respectively. The dynamics are obtained by discretizing the following continuoustime dynamics.

The crane is modeled as cart-pole system [6]:

$$
\begin{aligned}
& \ddot{\theta}=\frac{M_{t} g \sin (\theta)-\cos (\theta)\left(F+M_{p} l \dot{\theta}^{2} \sin (\theta)\right)}{l\left(4 / 3 M_{t}-M_{p} \cos ^{2}(\theta)\right)}=f_{1}^{c}(\theta, \dot{\theta}, F) \\
& \ddot{z}=\frac{F+M_{p} l \dot{\theta}^{2} \sin (\theta)-M_{p} l \ddot{\theta} \cos (\theta)}{M_{t}}=f_{2}^{c}(\theta, \dot{\theta}, F),
\end{aligned}
$$

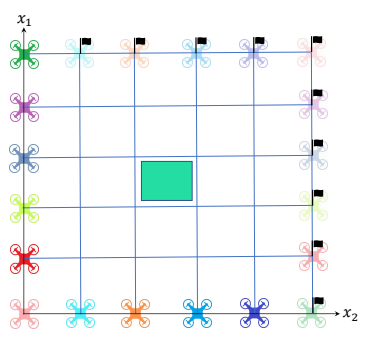

Figure 8: The mission map for the multi-drone path planning example

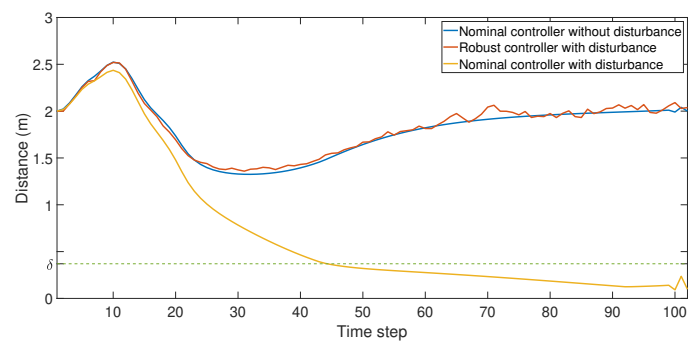

Figure 9: Performance of open-loop and feedback controllers in regulating distance between two selected drones for disturbance-free and perturbed situations for the multidrone path planning example

where $g=-9.8 \mathrm{~m} / \mathrm{s}^{2}$ is the acceleration of gravity, $M_{c}=1 \mathrm{~kg}$ is the cart mass, $M_{p}=0.1 \mathrm{~kg}$ is the pole mass, $M_{t}=M_{c}+M_{p}$ denotes the total mass, and $l=0.5 \mathrm{~m}$ is the half-pole length. Further, the cart's position, the pole's angle, and input force to the cart are denoted by $x_{1}^{(1)}=z, x_{3}^{(1)}=\theta$, and $u^{(1)}=F$, respectively. The continuous-time dynamics of the crane is of the following form:

$$
f^{c}\left(x^{(1)}(t), u^{(1)}(t)\right):=\left[\begin{array}{c}
\dot{x}_{1}^{(1)} \\
\dot{x}_{2}^{(1)} \\
\dot{x}_{3}^{(1)} \\
\dot{x}_{4}^{(1)}
\end{array}\right]=\left[\begin{array}{c}
\dot{z} \\
\ddot{z} \\
\dot{\theta} \\
\ddot{\theta}
\end{array}\right]=\left[\begin{array}{c}
x_{2}^{(1)} \\
f_{1}^{c}\left(x_{3}^{(1)}, x_{4}^{(1)}, u^{(1)}\right) \\
x_{4}^{(1)} \\
f_{2}^{c}\left(x_{3}^{(1)}, x_{4}^{(1)}, u^{(1)}\right)
\end{array}\right] .
$$

The vehicle's continuous-time dynamics takes the form of

$$
f^{l}\left(x^{(2)}(t), u^{(2)}(t)\right)=\left[\begin{array}{l}
\dot{x}_{1}^{(2)} \\
\dot{x}_{2}^{(2)}
\end{array}\right]=\left[\begin{array}{l}
x_{2}^{(2)} \\
u^{(2)}
\end{array}\right],
$$

where $x_{1}^{(2)}$ and $x_{2}^{(2)}$ denote the vehicle's position and speed, and $u^{(2)}$ represents the vehicle's control input (acceleration). On fixing the sampling time $\tau=0.1 \mathrm{~s}$, one can derive $f_{\tau}^{c}$ and $f_{\tau}^{l}$. For the crane, the disturbance set and robustness margin are chosen as $\left|W^{1}\right| \leq$ $(0,0.05,0,0)$ and $\varepsilon^{1}=(0.135,0.385,0.176,0.768)$. Similarly, for the vehicle, disturbance set and robustness margin are chosen as $\left|W^{2}\right| \leq(0,0.1)$ and $\varepsilon^{2}=(0.08,0.12)$.

There is no obstacle for this example and for minimum distance between the crane and the vehicle we choose $\delta=0.035 \mathrm{~m}$. Fixing the horizon length to $T=70$, ALTRO was capable of generating a valid nominal trajectory in 0.65 seconds. Fig. 2 (left) demonstrates 


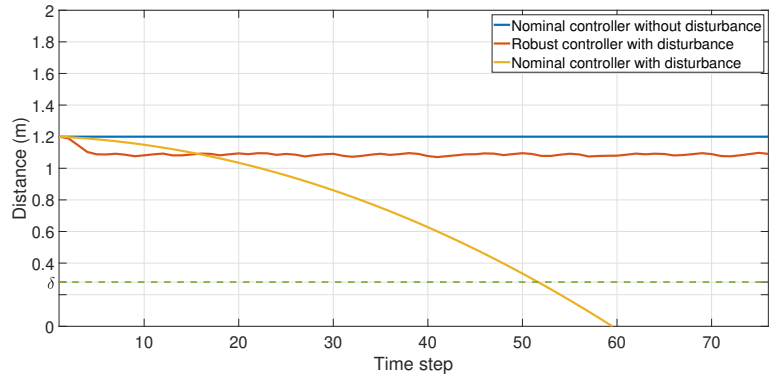

Figure 10: Comparison of open-loop and feedback controllers for the lane merging example

snapshots of the produced trajectory. As before, under the nominal open-loop controllers, applying (constant) additive disturbance $W=$ $(0,0.05,0,0)$ (to the cart-pole system) causes a collision between the crane and the vehicle before the end of the mission (Fig. 2 (right)).

In the next step, we use SCOTS in order to compute a feedback controller tolerating disturbances. We choose state and input spaces for the crane to be $X^{1}=[-0.195,5.49] \times$ $[-1.99,4.37] \times[1.20,4.68] \times[-5.44,5.28]$ and $U^{1}=[-7,7]$, respectively. For the vehicle, we set $X^{2}=[3,9] \times[-3,1.995]$ and $U^{2}=[-3,3]$. We choose state and input partition sizes $\eta_{X}^{1}=(0.015,0.035,0.016,0.064), \eta_{U}^{1}=0.2, \eta_{X}^{2}=(0.01,0.015)$ and $\eta_{U}^{2}=0.1$. Tab. 2 shows the run times and number of stateinput pairs corresponding to local and global $\mathrm{ABCD}$. As before, for the cart-pole model, global ABCD exceeds our 1.5 TB memory limit. Note that computing feedback controllers for the crane and vehicle takes 511 seconds and 0.3 seconds, respectively. The large difference is due to the difference in the size of transition systems for the two dynamics.

\section{A.3 Lane Merging}

The nominal dynamics for each of the vehicles is the same as the one for modeling drones (given in Sec. A.1). The disturbance set and robustness margin are chosen as $|W| \leq(0.03,0.03,0.03)$ and $\varepsilon=$ $(0.16,0.16,0.16)$. For collision and obstacle avoidance, we choose $\delta=0.37 \mathrm{~m}$. The horizon length is fixed to $T=110$. Given these settings, ALTRO generates a valid nominal trajectory in 89.02 seconds. Next, we use ABCD in order to compute feedback controllers tolerating additive disturbance $W$. We choose state and input spaces for each vehicle's model to be $X=[-0.5,15] \times[0.1,7.4] \times[-1,0.4]$ and $U=[-0.9,3] \times[-2.1,2.1]$, respectively. State and input partition sizes are chosen as $\eta_{X}=(0.02,0.02,0.02)$ and $\eta_{U}=(0.3,0.15)$. Tab. 2 shows run times and number of state-input pairs corresponding to local and global $\mathrm{ABCD}$. For $N>1$, memory requirement for global ABCD exceeds memory limits. Fig. 6 demonstrates snapshots of one sample trajectory when feedback controllers are employed under the presence of disturbance. It should be noticed that using ALTRO alone would not provide guarantee against bounded disturbance. Fig. 10 illustrates the fact that open-loop controller fails in keeping one of the vehicles away from the road's sides under perturbed situation when constant additive disturbance vector $(-0.03,0.03,-0.03)$ is being applied throughout the whole horizon.

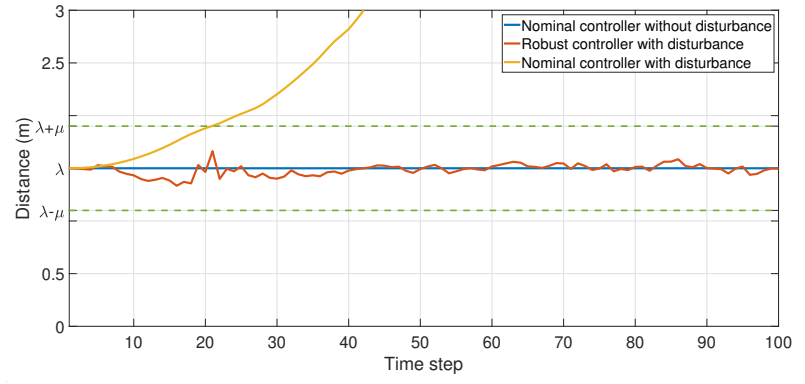

Figure 11: Comparison of open-loop and feedback controllers for the formation control example

In contrast, employing a feedback controller results in successful lane merging.

\section{A.4 Multi-drone formation control}

In the multi-drone formation control case study, the nominal dynamics for each of the drones is the same as that in Sec. A.1. The disturbance set and robustness margin are chosen as $|W| \leq$ $(0.03,0.03,0.03)$ and $\varepsilon=(0.24,0.24,0.24)$. Distance between each pair of drones positioned at the diamond's vertices is set to be $\lambda^{i, j}=\frac{3 \sqrt{2}}{2} \mathrm{~m}$ for $i, j \in\{1,2,3,4\}$, while the drone positioned at the center is supposed to keep distance $\lambda^{5, j}=1.5 \mathrm{~m}$ for $j \in\{1,2,3,4\}$. Setting the minimum distance for obstacle avoidance to $\delta=0.4$ $\mathrm{m}$ and horizon length $T=100$, ALTRO finds a valid solution over the product system with 15 state and 10 input variables within 114.3 seconds. Next, we synthesize local controllers for every drone such that the specifications hold for the perturbed models with $\mu=0.5 \mathrm{~m}$. We consider state and input spaces to be $X=[-2,17] \times[-2,17] \times[0.6,1.6]$ and $U=[-0.9,4.8] \times[-3,3]$, respectively. We select $\eta_{X}=(0.03,0.03,0.03)$ and $\eta_{U}=(0.3,0.15)$. Tab. 2 shows the run times and number of state-input pairs corresponding to local and global ABCD. Already for two drones, the memory requirement for global $\mathrm{ABCD}$ exceeds the available memory of 1.5 TB of RAM. Fig. 7 illustrates four sequential frames of a sample perturbed trajectory generated by employing feedback controllers. Notice that both relative position and orientation between drones are kept (almost) constant throughout the journey. On the other hand, using ALTRO alone would not provide guarantee against bounded disturbance. Fig. 11 illustrates performance of open-loop and feedback controllers on regulating distance between two specific drones with and without disturbances. As expected, in the absence of disturbances, the open-loop controllers suffice and the distance between the two drones (shown in solid blue) does not go below the threshold line (showed as the dotted line). However, when constant additive disturbance vectors $(0,0.03,0.03)$ and $(0,-0.03,-0.03)$ are being applied to the two drones throughout the whole horizon, open-loop controller fails, whereas the feedback controller is still capable of maintaining distance above the given threshold. 